\title{
Editorial for the Special Issue: "Recent developments in energy modeling and management"
}

\author{
Spiros Papaefthimiou ${ }^{1}$. Constantin Zopounidis ${ }^{1,2}$. \\ Kostas Andriosopoulos ${ }^{3}$
}

Received: 12 December 2015 / Accepted: 14 December 2015 / Published online: 4 January 2016 (C) Springer-Verlag Berlin Heidelberg 2015

Global demand for energy is increasing, while the concerns for related environmental burdens and climate change effects are continuously increasing. Issues involving fuel prices volatility, markets deregulation, geopolitical instabilities and renewables penetration in the grid are in almost all energy related agendas. This explosive mixture sets demanding prerequisites for people dealing with energy related issues: specially trained experts are necessary along with people with different backgrounds (namely from industry and universities), technicians and modelers. The challenge is not only scientific or technological but also managerial. Apart from important policy decisions in the energy sector, a global goal to sustain energy supplies, improve efficiency and develop new sustainable energy sources will demand technological innovations, capital funding and expertize in various aspects of management (i.e. energy related, human resources, supply chain, etc.). Moreover, the application of operation research methods is important in striving to resolve the conflicts between the various competing goals in pursuit of economic prosperity, environmental quality, social equity and technological efficiency.

Some of the papers included in this Special Issue have been presented at the 2nd International Conference of the Research Centre for Energy Management (RCEM) which took place on 22-24 of May, 2014 in Chania, Crete. The Conference was organized by the Technical University of Crete, in collaboration with the RCEM at ESCP Europe Business School and aimed to provide a forum for researchers and practitioners

Spiros Papaefthimiou

spapaefthimiou@dpem.tuc.gr

1 School of Production Engineering and Management, Technical University of Crete, Chania, Greece

2 Audencia Group, School of Management, Nantes, France

3 ESCP Europe Business School, London, UK 
to exchange ideas and to present new research results on the theory and modern practice of modelling, risk management, asset pricing and energy management, with also a focus on commodities modelling and regulations. Hence, this Special Issue comprises eight papers and contains an eclectic collection of research ideas into a number of different facets of energy markets, including high-quality papers on aspects of electricity markets, oil prices forecasting, and renewable technologies.

The first paper by Sotiriadis et al. examines price and volatility interrelationships in five (French, German, Belgian, Dutch as well as the Nord Pool Spot) European wholesale spot electricity markets. A novel VAR model with dummy variables was developed to model the conditional mean price, while the CCC-MGARCH model and a DCC-MGARCH model were used to model volatility. The results suggest that evidence of market integration, as measured by cross-mean spillovers and conditional correlation, do exist in the electricity markets under examination. Nevertheless, they also indicate that the Central-Western European region electricity markets are stronger integrated, while, on the other hand, weaker integration is observed between them and the Nordic electricity market. These findings are attributed to the fact that physical interconnection capacity is not sufficient for the electricity markets to become fully integrated.

In the second paper, authored by Sehgal et al. artificial intelligent methods are used for oil price forecasting as an alternate approach to conventional techniques. The following price forecasting techniques have been covered: (i) artificial neural network, (ii) support vector machine, (iii) wavelet, (iv) genetic algorithm, and (v) hybrid systems. In order to investigate the state of artificial intelligent models for oil price forecasting, thirty five research papers (published during 2001-2013) had been reviewed in form of table (for ease of comparison) based on the following parameters: (a) input variables, (b) input variables selection method, (c) data characteristics (d) forecasting accuracy and (e) model architecture. This review reveals procedure of AI methods used in complex oil price related studies. The review further extended above overview into discussions regarding specific shortcomings that are associated with feature selection for designing input vector, and then concluded with future insight on improving the current state-of-the-art technology.

Atsalakis et al. in their paper present a novel technique to forecast the price trend of 25 different commodities, listed on international markets, using a neuro-fuzzy controller. The forecasting system is based on two independent adaptive neural fuzzy inference systems (ANFISs) that form an inverse controller for each commodity. The ANFIS controller belongs to direct control and is based on inverse learning, also known as general learning. Daily data return sets have been used to learn and evaluate the proposed system, while the forecast accuracy of the proposed technique is evaluated by out-of-sample tests. The results are very encouraging, showing high accuracy of the hit rate reaching $68.33 \%$ and a notable superiority of the return on equity when compared with the buy and hold strategy.

In the fourth paper, Bienstock et al. present a rigorous analysis of the evolution of the temperature of a power line under stochastic exogenous factors such as ambient temperature. A solution to the resulting stochastic heat equation is presented alongside with a number of control algorithms designed to maximize delivered power under 
chance constraints used to limit the probability that a line exceeds its critical temperature.

In the next paper, Tiba et al. examine the four-way interrelationship between renewable energy, environment, foreign trade and growth using simultaneous-equation panel data models for 24 middle- and high-income countries over the period 1990-2011. Their findings show that, for the high-income countries, there is a bidirectional causality between renewable energy and growth, between $\mathrm{CO}_{2}$ emissions and economic growth, between foreign trade and growth and between renewable energy and $\mathrm{CO}_{2}$ emissions. However, there is a unidirectional causality between foreign trade and renewable energy and between emissions and trade. In the case of middle-income countries, there is also a bidirectional causality between renewable energy and growth, between $\mathrm{CO}_{2}$ emissions and growth, between trade and growth, between trade and renewable energy and between $\mathrm{CO}_{2}$ emissions and trade. On the other hand, there is a unidirectional causality, running from renewable energy to $\mathrm{CO}_{2}$ emissions. Understanding these controversial scenarios is prerequisite to reaching an international agreement on climate change in order to build sound economic policies and improving the environmental quality to sustain economic development.

Souliotis et al. present the technology of Integrated Collector Storage Solar Water Heaters (ICSSWH) which convert solar radiation directly into heat at an appreciable conversion rate and in many cases using concentrating means. These systems are compact, aesthetically attractive and reasonable in construction and can reduce the environmental impact up to $40 \%$. They also have high collection efficiency factor and energy saving potential. Despite of many advantages, ICS solar water heaters suffer from high thermal losses in the night/overcast sky conditions. In this article, authors discuss the recently developed new and improved ICS designs and strategies used for reducing thermal losses from such devices, especially in non-collection period. The systems have been evaluated based on a followed categorization to non-concentrating, concentrating and systems with phase change materials.

In the seventh paper, Katsigiannis et al. present a software tool that has been developed for optimal configuration of hybrid power systems. These systems can be either interconnected to the main power grid or operated autonomously, and may contain a variety of components, including dispatchable generators (e.g., diesel generators, microturbines, biogas generators), non-dispatchable renewable energy technologies (e.g., wind turbines, photovoltaics), batteries, converters and dump loads. A software tool that optimizes such systems has been developed in MATLAB, using a combination of genetic algorithms and tabu search. The optimal configuration is expressed in terms of minimum cost of electricity (in $€ / \mathrm{kWh}$ ), taking into account operational and component size constraints. The needed input includes weather data (e.g., solar, wind, and temperature time-series), load data, system components data, and general parameters (e.g., project lifetime, discount rate). As a case study, in this paper the tool is used for evaluating an autonomous hybrid power system that includes renewable energy technologies in Chania region, Crete. Moreover, the performance of the tool is investigated for seven additional scenarios of the case study, via sensitivity analysis, studying the effect on the results of the uncertainty of weather and cost data.

The last paper, Pati et al. deals with maximum power point tracking (MPPT) of a photovoltaic (PV) system under partially shaded conditions and active power support to 
the grid with power quality improvement at the point of common coupling (PCC). The overall system also provides an efficient power management among the various power sources, i.e., photovoltaic system, battery, and grid. The proposed MPPT algorithm is based on two important aspects, i.e., power versus voltage characteristic curve of the PV array under normal as well as partial shading conditions and a modified Fibonacci search algorithm. The PV power conditioning system is connected to the grid through an inverter and a DC-link capacitor. The inverter works both as a DC-AC converter for the PV system and as an active power filter for compensation of harmonics and reactive power. This system is cost-effective as a single inverter is used for both power quality improvement and power flow control from the PV system to the grid. Under unavailability of main AC supply, the operation is switched over to the standalone mode in which the PV system along with the battery is used to meet the load power demand. The performances of the above system are verified by simulation studies in MATLAB/SIMULINK environment and on a real time experimental prototype system using dSPACE controller.

The authors of the above mentioned papers, offer their insights into a number of issues, but also provide leads to further interesting research, so we are certain that you will enjoy reading them. Closing this editorial, we should express our sincere thanks to all authors whose contributions have been essential in completing this rich and high quality special issue. We should also acknowledge the support of all reviewers who devoted considerable time to provide critical evaluations, insightful comments, and constructive suggestions for all submitted papers. Without their help it would be impossible to achieve this issue's high standards. Finally, we are grateful to the Editorial team of Energy Systems, for the acceptance of our proposal for this special issue and their continuous support and guidance throughout the editorial process and the handling of the submitted papers. 